\title{
Orthogonal Method for Linear Systems. Preconditioning
}

\author{
Henar Herrero ${ }^{1}$, Enrique Castillo ${ }^{1,2}$ and Rosa Eva Pruneda ${ }^{1}$ \\ ${ }^{1}$ Departamento de Matemáticas, Universidad de Castilla-La Mancha, \\ 13071 Ciudad Real, Spain. \\ 2 Departamento de Matemática Aplicada y Ciencias de la Computacin, \\ Universidad de Cantabria, Santander, Spain.
}

\begin{abstract}
The complexity of an orthogonal method for solving linear systems of equations is discussed. One of the advantages of the orthogonal method is that if some equations of the initial linear system are modified, the solution of the resulting system can be easily updated with a few extra operations, if the solution of the initial linear system is used. The advantages of this procedure for this updating problem are compared with other alternative methods. Finally, a technique for reducing the condition number, based on this method, is introduced.
\end{abstract}

\section{Introduction}

Castillo et al [1] have introduced a pivoting transformation for obtaining the orthogonal of a given linear subspace. This method is applied to solve a long list of problems in linear algebra including systems of linear equations. Nowadays interest is focussed on iterative methods [2], [3], [4], [5] because they are more suitable for large systems. However, they present some difficulties such as conditioning, and for some problems a direct method can be more satisfactory. The direct methods arising from this transformation have complexity identical to that associated with the Gaussian elimination method (see Castillo et al [1]). However, they are specially suitable for updating solutions when changes in rows, columns, or variables are done. In fact, when changing a row, column or variable, a single step of the process allows obtaining (updating) the new solution, whithout the need of starting from scratch again. Therefore a drastic reduction in computational effort is obtained.

The paper is structured as follows. In Section 2 the pivoting transformation is introduced. In Section 3 this transformation is applied to find the general solution of a linear system of equations. In Section 4 the main advantage of the method, i.e., the updating of solutions, is analyzed. In Section 5 the complexity of the method related to its updating facilities is studied. Finally, in Section 6 an strategy to reduce the condition number based on this orthogonal procedure is given. 


\section{Pivoting Transformation}

The main tool to be used in this paper consists of the so called pivoting transformation, which transforms a set of vectors $\mathbf{V}^{j}=\left\{\mathbf{v}_{1}^{j}, \ldots, \mathbf{v}_{n}^{j}\right\}$ into another set of vectors $\mathbf{V}^{j+1}=\left\{\mathbf{v}_{1}^{j+1}, \ldots, \mathbf{v}_{n}^{j+1}\right\}$ by

$$
\mathbf{v}_{k}^{j+1}=\left\{\begin{array}{l}
\mathbf{v}_{k}^{j} / t_{j}^{j} ; \quad \text { if } k=j, \\
\mathbf{v}_{k}^{j}-\frac{t_{k}^{j}}{t_{j}^{j}} \mathbf{v}_{j}^{j} ; \text { if } k \neq j,
\end{array}\right.
$$

where $t_{j}^{j} \neq 0$ and $t_{k}^{j} ; k \neq j$ are arbitrary real numbers. Note that the set $\left\{t_{1}^{j}, t_{2}^{j}, \cdots, t_{n}^{j}\right\}$ defines this transformation. In what follows the vectors above are considered as the columns of a matrix $\mathbf{V}^{j}$.

This transformation can be formulated in matrix form as follows. Given a matrix $\mathbf{V}^{j}=\left[\mathbf{v}_{1}^{j}, \ldots, \mathbf{v}_{n}^{j}\right]$, where $\mathbf{v}_{i}^{j}, i=1, \ldots, n$, are column vectors, a new matrix $\mathbf{V}^{j+1}$ is defined via

$$
\mathbf{V}^{j+1}=\mathbf{V}^{j} \mathbf{M}_{j}^{-1}
$$

where $\mathbf{M}_{j}^{-1}$ is the inverse of the matrix

$$
\mathbf{M}_{j}=\left(\mathbf{e}_{1}, \ldots, \mathbf{e}_{j-1}, \mathbf{t}_{j}, \mathbf{e}_{j+1}, \ldots, \mathbf{e}_{n}\right)^{T},
$$

and $\mathbf{e}_{i}$ is the $i$ th column of the identity matrix. In this paper this transformation is asociated with a vector $\mathbf{a}_{j}$, so that the transpose of $\mathbf{t}_{j}$ is defined by

$$
\mathbf{t}_{j}^{T}=\mathbf{a}_{j}^{T} \mathbf{V}^{j} .
$$

Since $t_{j}^{j} \neq 0$, the matrix $\mathbf{M}_{j}$ is invertible. It can be proved that $\mathbf{M}_{j}^{-1}$ is the identity matrix with its $j$ th row replaced by

$$
\mathbf{t}_{j}^{*}=\frac{1}{t_{j}^{j}}\left(-t_{1}^{j}, \ldots,-t_{j-1}^{j}, 1,-t_{j+1}^{j}, \ldots,-t_{n}^{j}\right) .
$$

This transformation is used in well-known methods, such as the Gaussian elimination method. However, different selections of the $t$-values lead to completely different results. In this paper this selection is based on the concept of orthogonality, and a sequence of $m$ transformations associated with a set of vectors $\left\{\mathbf{a}_{1}, \ldots, \mathbf{a}_{m}\right\}$ is assumed.

The main properties of this pivoting transformation can be summarized as follows (see [6]):

1. Given a matrix $\mathbf{V}$, the pivoting transformation transforms its columns without changing the linear subspace they generate, i.e., $\mathcal{L}\left(\mathbf{V}^{j}\right) \equiv \mathcal{L}\left(\mathbf{V}^{j+1}\right)$. 
2. The pivoting process (2) with the pivoting strategy (4) leads to the orthogonal decomposition of the linear subspace generated by the columns of $\mathbf{V}^{j}$ with respect to vector $\mathbf{a}_{j}$. Let $\mathbf{a}_{j} \neq \mathbf{0}$ be a vector and let $t_{k}^{j}=\mathbf{a}_{j}^{T} \mathbf{v}_{k}^{j} ; k=$ $1, \ldots, n$. If $t_{j}^{j} \neq 0$, then

$$
\mathbf{a}_{j}^{T} \mathbf{V}^{j+1}=\mathbf{e}_{j}^{T}
$$

In addition, the linear subspace orthogonal to $\mathbf{a}_{j}$ in $\mathcal{L}\left(\mathbf{V}^{j}\right)$ is

$$
\left\{\mathbf{v} \in \mathcal{L}\left(\mathbf{V}^{j}\right) \mid \mathbf{a}_{j}^{T} \mathbf{v}=0\right\}=\mathcal{L}\left(\mathbf{v}_{1}^{j+1}, \ldots, \mathbf{v}_{j-1}^{j+1}, \mathbf{v}_{j+1}^{j+1}, \ldots, \mathbf{v}_{n}^{j+1}\right)
$$

and its complement is $\mathcal{L}\left(\mathbf{v}_{j}^{j+1}\right)$. In other words, the transformation (2) gives the generators of the linear subspace orthogonal to $\mathbf{a}_{j}$ and the generators of its complement.

3. Let $\mathcal{L}\left\{\mathbf{a}_{1}, \ldots, \mathbf{a}_{n}\right\}$ be a linear subspace. Then, the pivoting transformation (2) can be sequentially used to obtain the orthogonal set to $\mathcal{L}\left\{\mathbf{a}_{1}, \ldots, \mathbf{a}_{n}\right\}$ in a given subspace $\mathcal{L}\left(\mathbf{V}^{1}\right)$. Let $t_{i}^{j}$ be the dot product of $\mathbf{a}_{j}$ and $\mathbf{v}_{i}^{j}$. Then assuming, without loss of generality, that $t_{j}^{j} \neq 0$, the following is obtained

$$
\begin{aligned}
\mathcal{L}\left(\mathbf{V}^{j}\right) & =\mathcal{L}\left(\mathbf{v}_{1}^{j}-\frac{t_{1}^{j}}{t_{q}^{j}} \mathbf{v}_{q}^{j}, \ldots, \mathbf{v}_{q}^{j}, \ldots, \mathbf{v}_{n}^{j}-\frac{t_{n}^{j}}{t_{q}^{j}} \mathbf{v}_{q}^{j}\right) \\
& =\mathcal{L}\left(\mathbf{v}_{1}^{j+1}, \ldots, \mathbf{v}_{n}^{j+1}\right)=\mathcal{L}\left(\mathbf{V}^{j+1}\right),
\end{aligned}
$$

and

$\mathcal{L}\left(\mathbf{a}_{1}, \ldots, \mathbf{a}_{j}\right)^{\perp} \equiv\left\{\mathbf{v} \in \mathcal{L}\left(\mathbf{V}^{1}\right) \mid \mathbf{a}_{1}^{T} \mathbf{v}=0, \ldots, \mathbf{a}_{j}^{T} \mathbf{v}=0\right\}=\mathcal{L}\left(\mathbf{v}_{j+1}^{j+1}, \ldots, \mathbf{v}_{n}^{j+1}\right)$.

In addition, we have

$$
\mathbf{a}_{r}^{T} \mathbf{v}_{k}^{j+1}=\delta_{r k} ; \forall r \leq j ; \forall j
$$

where $\delta_{r k}$ are the Kronecker deltas.

4. The linear subspace orthogonal to the linear subspace generated by vector $\mathbf{a}_{j}$ is the linear space generated by the columns of $\mathbf{V}^{k}$; for any $k \geq j+1$ with the exception of its pivot column, and its complement is the linear space generated by this pivot column of $\mathbf{V}^{k}$.

5. The linear subspace, in the linear subspace generated by the columns of $\mathbf{V}^{1}$, orthogonal to the linear subspace generated by any subset $W=\left\{\mathbf{a}_{k} \mid k \in K\right\}$ is the linear subspace generated by the columns of $\mathbf{V}^{\ell}, \ell>\max _{k \in K}$ with the exception of all pivot columns associated with the vectors in $W$, and its complement is the linear subspace generated by the columns of $\mathbf{V}^{\ell}, \ell>$ $\max _{k \in K}$ which are their pivot columns.

The following theorem shows how the orthogonal transformation can be used, not only to detect when a vector is a linear combination of previous vectors used in the pivoting process, but to obtain the coefficients of such combination. 
Theorem 1 (Linear combination). Let $\mathcal{L}\left\{\mathbf{a}_{1}, \ldots, \mathbf{a}_{n}\right\}$ be a linear subspace. Applying sequentially the pivoting transformation (2), if $t_{k}^{j}=0$ for all $k=$ $j, \ldots, n$, then the $\mathbf{a}_{j}$ vector is a linear combination of previous vectors used in the process,

$$
\mathbf{a}_{j}=\rho_{1} \mathbf{a}_{1}+\ldots+\rho_{q-1} \mathbf{a}_{j-1},
$$

where $\rho_{i}=t_{i}^{j}=\mathbf{a}_{j} \bullet \mathbf{v}_{i}^{j}$, and $\mathbf{v}_{i}^{j}$ is the corresponding pivot column associated with the vector $\mathbf{a}_{i}$, for all $i=1, \ldots, j-1$.

Proof. If $t_{k}^{j}=0$ for all $k=j, \ldots, n$,

$$
\mathbf{a}_{j} \in \mathcal{L}^{\perp}\left\{\mathbf{a}_{1}, \ldots, \mathbf{a}_{j-1}\right\}^{\perp} \equiv \mathcal{L}\left\{\mathbf{a}_{1}, \ldots, \mathbf{a}_{j-1}\right\},
$$

then $\exists \rho_{1}, \ldots, \rho_{j-1}$, such that $\mathbf{a}_{j}=\rho_{1} \mathbf{a}_{1}+\ldots+\rho_{j-1} \mathbf{a}_{j-1}$. For $i=1, \ldots, n$, we calculate the dot product

$$
\mathbf{a}_{j} \bullet \mathbf{v}_{i}^{j}=\left(\rho_{1} \mathbf{a}_{1}+\ldots+\rho_{q-1} \mathbf{a}_{q-1}\right) \bullet \mathbf{v}_{i}^{j}=\rho_{1}\left(\mathbf{a}_{1} \bullet \mathbf{v}_{i}^{j}\right)+\ldots+\rho_{j-1}\left(\mathbf{a}_{j-1} \bullet \mathbf{v}_{i}^{j}\right)
$$

and using property 4 of Section $2, \mathbf{a}_{i} \bullet \mathbf{v}_{i}^{j}=1$ and $\mathbf{a}_{k} \bullet \mathbf{v}_{i}^{j}=0$ for all $k \neq i, k=$ $1, \ldots, j-1$, we obtain $\mathbf{a}_{j} \bullet \mathbf{v}_{i}^{j}=\rho_{i}$.

\section{Solving a linear system of equations}

Consider now the complete system of linear equations $\mathbf{A x}=\mathbf{b}$ :

$$
\begin{array}{ccc}
a_{11} x_{1}+a_{12} x_{2}+\cdots+a_{1 n} x_{n}= & b_{1} \\
a_{21} x_{1}+a_{22} x_{2}+\cdots+a_{2 n} x_{n}= & b_{2} \\
\ldots & \cdots & \cdots \\
\cdots & \cdots & \cdots \\
a_{m 1} x_{1}+a_{m 2} x_{2}+\cdots+a_{m n} x_{n}= & b_{m}
\end{array}
$$

Adding the artificial variable $x_{n+1}$, it can be written as:

$$
\begin{aligned}
& a_{11} x_{1}+a_{12} x_{2}+\cdots+a_{1 n} x_{n}-b_{1} x_{n+1}=0 \\
& a_{21} x_{1}+a_{22} x_{2}+\cdots+a_{2 n} x_{n}-b_{2} x_{n+1}=0 \\
& \begin{array}{llllllll}
\ldots & \ldots & \ldots & \ldots & \ldots & \ldots
\end{array} \\
& a_{m 1} x_{1}+a_{m 2} x_{2}+\cdots+a_{m n} x_{n}-b_{m} x_{n+1}=0 \\
& a_{m 1} x_{1}+a_{m 2} x_{2}+\cdots+a_{m n} x_{n}-b_{m} x_{n+1}=0 \\
& x_{n+1}=1
\end{aligned}
$$

System (11) can be written as:

$$
\begin{array}{cc}
\left(a_{11}, \cdots, a_{1 n},-b_{1}\right)\left(x_{1}, \cdots, x_{n}, x_{n+1}\right)^{T}= & 0 \\
\left(a_{21}, \cdots, a_{2 n},-b_{2}\right)\left(x_{1}, \cdots, x_{n}, x_{n+1}\right)^{T}= & 0 \\
\cdots \cdots \cdots \cdots \cdots \cdots \cdots \cdots \cdots \cdots \cdots \cdots & \cdots \\
\left(a_{m 1}, \cdots, a_{m n},-b_{m}\right)\left(x_{1}, \cdots, x_{n}, x_{n+1}\right)^{T}= & 0
\end{array}
$$

Expression (12) shows that $\left(x_{1}, \ldots, x_{n}, x_{n+1}\right)^{T}$ is orthogonal to the set of vectors:

$$
\left\{\left(a_{11}, \ldots, a_{1 n},-b_{1}\right)^{T},\left(a_{21}, \ldots, a_{2 n},-b_{2}\right)^{T}, \ldots,\left(a_{m 1}, \ldots, a_{m n},-b_{m}\right)^{T}\right\} .
$$


Then, it is clear that the solution of (11), is the orthogonal complement of the linear subspace generated by the rows of matrix

$$
\overline{\mathbf{A}}=(\mathbf{A} \mid-\mathbf{b}),
$$

i.e., the column $\mathbf{- b}$ is added to the matrix $\mathbf{A}$ :

$$
\mathcal{L}\left\{\left(a_{11}, \ldots, a_{1 n},-b_{1}\right)^{T},\left(a_{21}, \ldots, a_{2 n},-b_{2}\right)^{T} \ldots,\left(a_{m 1}, \ldots, a_{m n},-b_{m}\right)^{T}\right\}^{\perp}
$$

Thus, the solution of (10) is the projection on $\mathbb{R}^{n}$ of the intersection of the orthogonal complement of the linear subspace generated by

$$
\left\{\left(a_{11}, \ldots, a_{1 n},-b_{1}\right)^{T},\left(a_{21}, \ldots, a_{2 n},-b_{2}\right)^{T}, \ldots,\left(a_{m 1}, \ldots, a_{m n},-b_{m}\right)^{T}\right\} .
$$

and the set $\left\{\mathbf{x} \mid x_{n+1}=1\right\}$.

To solve system (12) we apply the orthogonal algorithm to the $\mathbf{A}$ rows with $\mathbf{V}^{1} \equiv \mathcal{L}\left\{\mathbf{v}_{1}^{1}, \ldots, \mathbf{v}_{n+1}^{1}\right\}$ where $\mathbf{v}_{i}^{1}=\mathbf{e}_{i} ; i=1, \ldots, n+1$, and $\mathbf{e}_{i}$ is the vector with all zeroes except for the $i$ th component, which is one.

If we consider the $j$-th equation of system (11), i.e., $\mathbf{a}_{j} \mathbf{x}=\mathbf{b}_{j}$, after pivoting with the corresponding associated vector, we can obtain the solution of this equation, $\mathbf{X}^{j}$,

$$
\mathbf{X}^{j} \equiv\left\{\mathbf{x} \in \mathbf{V}^{k} \mid x_{n+1}=1 \wedge \mathbf{a}_{j}^{T} \bullet \mathbf{x}=0, k=j+1, \ldots m\right\} .
$$

In fact, we consider the solution generated by the columns of the corresponding table except the column used as pivot (see Section 2, property 5), and we impose the condition $x_{n+1}=1$.

After this sequential process we have $\mathbf{X}^{m} \equiv \mathcal{L}\left\{\mathbf{v}_{1}^{m}, \ldots, \mathbf{v}_{n_{m}}^{m}\right\}$, and then the solution of the initial system becomes

$$
\hat{\mathbf{v}}_{n_{m}}^{m}+\mathcal{L}\left\{\hat{\mathbf{v}}_{1}^{m}, \ldots, \hat{\mathbf{v}}_{n_{m}-1}^{m}\right\},
$$

where $\hat{\mathbf{v}}$ is the vector obtained from $\mathbf{v}$ by removing its last component (see table 2 for an example).

When $\mathcal{L}\left(\hat{\mathbf{v}}_{1}^{m}, \ldots, \hat{\mathbf{v}}_{n_{m}-1}^{m}\right)$ degenerates to the empty set, we have uniqueness of solution. For this to occur, we must have $m=n$ and $|\mathbf{A}| \neq 0$; that is, the coefficients matrix must be a square nonsingular matrix.

If we are interested in obtaining the solution of any subsystem, we will take the intersections of the corresponding solutions of each equation. Note that we have to keep the orthogonal and the complement set in each step for this process to be applied.

\section{Modifying equations}

Once the redundant equations have been detected in a system of linear equations, which is easy using the orthogonal method for solving the system (see Theorem 1 ), we can suppose without lost of generality, that the linear system is not 
redundant. In this section we show how to update the solution of this system after modifying one equation, with only one extra iteration of the orthogonal method.

Consider a not redundant initial linear system of equations $\mathbf{A x}=\mathbf{b}$, and use the proposed method to solve it, but keeping the complement spaces. If the $j$ th equation is modified, to obtain the new solution, the orthogonal subspace corresponding to the new equation is needed, instead of the orthogonal to the old equation. Since the orthogonal method can be started with any space $\mathbf{V}$, the last table of the initial process can be taken as the initial table for solving the modified system and the modified equation can be introduced to make the extra pivoting transformation. Using as pivot the pivot column associated with the modified row, and taking into account properties 1 and 4 from Section 2, the solution of the modified system is obtained.

\section{Complexity}

Castillo et al [1] have studied the number of operations required by the orthogonal method for solving a linear system of equations. The conclusion of this study is that when this method is used for obtaining the final solution of a linear system of equations and in each step the complement space is removed, the number of operations is the same as the number of operations required for the Gaussian elimination method, that is, around $2 n^{3} / 3$ operations (see [7]). The aim in this section is to study the number of operations of the complete process, keeping the complement space, in order to allow the updating of solutions after modifying an equation, and to compare this results with de Gaussian process.

The number of operations required to solve a linear system of equations with the Gaussian elimination method (see [8]) is $2 n^{3} / 3+\left(9 n^{2}-7 n\right) / 6$, and with the orthogonal method it is $\left(2 n^{3}-5 n+6\right) / 3$ (see Ref.[1]). However, the number of operations of the orthogonal method keeping the complement space is $2 n^{3}-n$.

When an equation is modified, an extra iteration is required to update the solution, which implies $4 n^{2}-2 n$ extra operations.

If several modifications in the original system are needed with the Gaussian elimination method we have to repeat the complete process each time to obtain the new solution. However, with the orthogonal method we need to make the complete process only once $\left(2 n^{3}-n\right.$ operations $)$ and one update for each modification $\left(4 n^{2}-2 n\right.$ operations). Then, the number of total required operations when the size of the system is $n$ and the number of updates is $k$, is

$$
2 n^{3}-n+\left[k\left(4 n^{2}-2 n\right)\right]
$$

for the orthogonal method, and

$$
k\left[(2 / 3) n^{3}+\left(9 n^{2}-7 n\right) / 6\right]
$$

for the Gaussian elimination method.

¿From these formulas we can conclude that for $k>3$ the number of operations required by the orthogonal method is smaller and then, it outperforms 
Table 1. Required number of products and sums for making an extra iteration of the orthogonal method.

\begin{tabular}{|c|c|c|}
\hline \multicolumn{3}{|c|}{ Extra iteration } \\
\hline & Products or divisions & Sums \\
\hline Dot Products & $n^{2}$ & $n(n-1)$ \\
\hline Normalization & $n$ & - \\
\hline Pivoting & $n(n-1)$ & $n(n-1)$ \\
\hline \hline Total & $2 n^{2}$ & $2\left(n^{2}-n\right)$ \\
\hline
\end{tabular}

the Gaussian elimination method. This fact can be useful for nonlinear problems solved by means of iterations on linear approaches, i.e., for Newton methods.

\section{Reducing the condition number}

In this section we propose a method for obtaining an equivalent system of linear equations with a very small condition number for a full matrix. This kind of matrices appears very often when using spectral methods for solving partial differential equations. The idea uses the procedure explained in section 3 for solving a linear system. It is based on detecting that the matrix is ill conditioned when several of the rows of the matrix $\mathbf{A}$ are in very close hyperplanes, i.e., when $\operatorname{tg}\left(\widehat{\mathbf{a}}_{i, \mathbf{a}_{j}}\right) \ll 1$. The solution consists of rotating one of the vectors, but keeping it into the same hyperplane $\mathcal{L}\left(\overline{\mathbf{a}}_{i}, \overline{\mathbf{a}}_{j}\right)$.

As an example, let $\overline{\mathbf{A}}$ be the matrix corresponding to the coefficient matrix whit the independent vector added of the following system:

$$
\begin{aligned}
& 0.832 x_{1}+0.448 x_{2}=1 \\
& 0.784 x_{1}+0.421 x_{2}=0 .
\end{aligned}
$$

Using the orthogonal method (pivoting transformation (1)), we can see in the second iteration of Table 2 , that $\mathcal{L}\left(\overline{\mathbf{a}}_{1}^{T}\right)^{\perp}=\mathcal{L}\left(\mathbf{v}_{2}^{1}, \mathbf{v}_{3}^{1}\right)$. When the vector $\overline{\mathbf{a}}_{2}^{T}$ in introduced into the pivoting process, it can be observed that it is almost orthogonal to $\mathbf{v}_{2}^{1}$, one of the generators of $\mathcal{L}\left(\overline{\mathbf{a}}_{1}\right)^{\perp}$, and this is the source of ill conditioning character $(K(\mathbf{A})=1755)$ of the given system.

To solve this problem the vector $\mathbf{a}_{2}$ can be replaced by a $\pi / 2$ rotation, $\mathbf{a}_{2}^{g}=$ $g_{\pi / 2}\left(\mathbf{a}_{2}\right)$, of $\mathbf{a}_{2}$ such that $\overline{\mathbf{a}}_{2}^{g}=\left(g_{\pi / 2}\left(\mathbf{a}_{2}\right), x_{23}\right) \in \mathcal{L}\left(\overline{\mathbf{a}}_{1}, \overline{\mathbf{a}}_{2}\right)$. In this way the linear space $\mathcal{L}\left(\overline{\mathbf{a}}_{1}, \overline{\mathbf{a}}_{2}\right)^{\perp}$ is the same as the linear space $\mathcal{L}\left(\overline{\mathbf{a}}_{1}, \overline{\mathbf{a}}_{2}^{g}\right)^{\perp}$.

To calculate $\overline{\mathbf{a}}_{2}^{g}$ the linear system $\mathbf{a}_{2}^{g}=\alpha \mathbf{a}_{1}+\beta \mathbf{a}_{2}$ is solved, and then $x_{23}$ is calculated as $x_{23}=-\alpha b_{1}-\beta b_{2}$. 
Table 2. Iterations for solving Eq. (16). Pivot columns are boldfaced.

\begin{tabular}{|c|c|c|c|c|c|c|c|}
\hline \multicolumn{4}{|c|}{ Iteration 1} & \multicolumn{4}{|c|}{ Iteration 2} \\
\hline$\overline{\mathbf{a}}_{1}^{T}$ & $\mathbf{v}_{1}$ & $\mathbf{v}_{2}$ & $\mathbf{v}_{3}$ & $\overline{\mathbf{a}}_{2}^{T}$ & $\mathbf{v}_{1}^{1}$ & $\mathbf{v}_{2}^{1}$ & $\mathbf{v}_{3}^{1}$ \\
\hline 0.832 & 1 & 0 & 0 & 0.784 & 1 & -0.538 & -1.202 \\
\hline 0.448 & $\mathbf{0}$ & 1 & 0 & 0.421 & 0 & 1 & 0 \\
\hline \multirow[t]{2}{*}{-1} & $\mathbf{0}$ & c & 1 & 0 & 0 & 0 & 1 \\
\hline & 0.832 & 0.448 & -1 & & 0.784 & $-7.92 \mathrm{e}-004$ & -0.942 \\
\hline & & & \multicolumn{3}{|c|}{ Output } & & \\
\hline & & & $\mathbf{v}_{1}^{2}$ & $\mathbf{v}_{2}^{2}$ & $\mathbf{v}_{3}^{2}$ & & \\
\hline & & & 1 & -0.538 & -438.542 & & \\
\hline & & & 0 & 1 & 816.667 & & \\
\hline & & & 0 & 0 & 1 & & \\
\hline
\end{tabular}

Table 3. Order of the condition number of the matrices equivalent to the Hilbert matrix of size $n=10$ rotated in the rows from $m$ to $n$.

\begin{tabular}{|c|c|c|c|c|c|c|c|c|}
\hline$m$ & 9 & 8 & 7 & 6 & 5 & 4 & 3 & 2 \\
\hline$K$ & $10^{10}$ & $10^{8}$ & $10^{7}$ & $10^{6}$ & $10^{4}$ & $10^{3}$ & $10^{2}$ & $10^{2}$ \\
\hline
\end{tabular}

If this technique is applied to the system (16), the new coefficient matrix is obtained

$$
\mathbf{A}^{\prime}=\left(\begin{array}{cc}
0.8320 & 0.4480 \\
-0.2261 & 0.4210
\end{array}\right)
$$

and $K\left(\mathbf{A}^{\prime}\right)=1.9775$, i.e., the condition number has been drastically reduced. The new independent term becomes $\mathbf{b}^{\prime}=\left(\begin{array}{ll}1 & 442.959\end{array}\right)^{T}$. It is straightforward to prove that the system $\mathbf{A}^{\prime} \mathbf{x}=\mathbf{b}^{\prime}$ is equivalent to the system (16).

In the case of a $n \times n$ system, to be sure that all the vectors are in different hyperplanes the following method can be applied:

Starting by $i=m(1<m<n)$ till $i=n$, the pairs $\left(a_{i i}, a_{i i+1}\right)$ are compared with the pairs $\left(a_{j i}, a_{j i+1}\right), j=i+1, \ldots, n$. If $\operatorname{tg}\left(\widehat{\mathbf{a}}_{i, \mathbf{a}}\right) \ll 1$ the corresponding vector $\left(a_{j i}, a_{j i+1}\right)$ is rotated to $\left(-a_{j i+1}^{2} / a_{j i}, a_{j i+1}\right)$ in such a way that the subspaces in $\mathbb{R}^{n+1}$ are kept, i.e.,

$$
\overline{\mathbf{a}}_{j}^{g}=\left(a_{j 1}, \ldots, a_{j i-1},-a_{j i+1}^{2} / a_{j i}, a_{j i+1}, x_{j i+2}, \ldots, x_{j n+1}\right) \in \mathcal{L}\left\{\overline{\mathbf{a}}_{1}, \overline{\mathbf{a}}_{2}, \ldots, \overline{\mathbf{a}}_{j}\right\}
$$


To calculate $\overline{\mathbf{a}}_{j}^{g}$ the following linear system, with unknowns $\alpha_{i}, i=1, \ldots, j$, is solved:

$$
\overline{\mathbf{a}}_{j}^{g t j}=\alpha_{1} \overline{\mathbf{a}}_{1}^{t j}+\ldots+\alpha_{j} \overline{\mathbf{a}}_{j}^{t j}
$$

where the superscript ${ }^{t j}$ means truncated up to the index $j$, and then

$$
x_{j k}=\alpha_{1} a_{1 k}+\ldots+\alpha_{j} a_{j k}
$$

In this way one can be sure that any two row vectors of the new matrix are in very different hyperplanes and the condition number is reduced. This fact can be observed in table $3: K(\mathbf{A})=O\left(10^{13}\right)$ for the Hilbert matrix, where it can be seen that if this technique is applied with increasing number of rows, the condition number reduces to $K=O\left(10^{2}\right)$ when all the rows are involved.

\section{References}

1. Castillo, E., Cobo, A., Jubete, F., Pruneda, R.E.:Orthogonal Sets and Polar Methods in Linear Algebra: Applications to Matrix Calculations, Systems of Equations and Inequalities, and Linear Programming. John Wiley and Sons, New York (1999)

2. Golub, G.H., van Loan, C.F.: Matrix Computations. Johns Hopkins University Press, London (1996)

3. Axelsson, O.: Iterative Solution Methods. Cambridge University Press, Cambridge (1996)

4. Kelley, C.T.: Iterative Methods for Linear and Nonlinear Equations. SIAM, Phyladelphia (1995)

5. Duff, I.S., Watson, G.A. (eds): The state of the art in numerical analysis. Oxford University Press, Oxford (1997)

6. Castillo, E., Cobo, A., Pruneda, R.E. and Castillo, C.: An Orthogonally-Based Pivoting Transformation of Matrices and Some Applications. SIAM Journal on Matrix Analysis and Applications 22 (2000) 666-681

7. Atkinson, K.E.: An Introduction to Numerical Analysis. Jonhn Wiley and Sons, New York (1978)

8. Infante del Río, J.A., Rey Cabezas, J.M.: Métodos numéricos. Teoría, problemas y prácticas con Matlab. Pirámide, Madrid (1999) 